\title{
How new fact boxes are explaining medical risk to millions
}

\author{
Smart "fact boxes" that communicate evidence based information on the benefits and harms of \\ drugs and health screening are being rolled out to millions of people in Europe. Gerd Gigerenzer \\ and Kai Kolpatzik report
}

\author{
Gerd Gigerenzer director ${ }^{1}$, Kai Kolpatzik head ${ }^{2}$
}

${ }^{1}$ Harding Center for Risk Literacy and Center for Adaptive Behavior and Cognition, Max Planck Institute for Human Development, Berlin, Germany; ${ }^{2}$ Department of Prevention, General Local Health Insurance Fund (AOK-Bundesverband), Berlin, Germany; Correspondence to: G Gigerenzer gigerenzer@mpib-berlin.mpg.de

\begin{abstract}
An alien investigating healthcare on Earth would be quite puzzled. We spend billions on clinical studies but fail to ensure that patients and physicians are communicated the results transparently. ${ }^{1}$ Instead they get persuasion, marketing, and, in some countries, misleading direct-to-consumer advertising. ${ }^{23}$

One tool to bridge this gap is the fact box. Originally designed for medication, it was introduced and tested by US researchers Lisa Schwartz and Steve Woloshin from Dartmouth College. Their studies showed that, unlike consumer drug adverts, which led to largely erroneous opinions, drug fact boxes substantially improved the understanding of the benefits and harms of drugs. ${ }^{4}$ Their research led to an ultimately doomed attempt in the US Senate to get the Food and Drug Administration to adopt the fact boxes. Action was taken elsewhere, however. Germany's largest health insurance provider, AOK, serving some 25 million people, has recently released 11 new fact boxes on topics such as MMR vaccination, flu vaccination for people aged over 60 , vitamin D supplements, and screening for ovarian cancer.
\end{abstract}

\section{How the boxes work}

The fact box on screening for ovarian cancer with ultrasonography and a serum cancer antigen 125 test (fig $1 \Downarrow$ ) shows how the format makes information clear and accessible. It starts by providing information about benefit under the summary line: "Annual ultrasound examinations do not reduce the risk of dying from ovarian cancer." Below that, the available facts are presented in absolute numbers. One design feature of a fact box is that the two alternatives-here, screening and no screening - are presented side by side so that the outcomes can easily be compared. Here, readers see that the death rate from ovarian cancer was three out of every 1000 women in each group. Thus, there is no evidence that this type of screening saves lives.

Further information is provided about the evidence under "annotations and references" (not shown). For example, it states that the PLCO study ${ }^{5}$ on which the information is based was conducted in the US and that it comprised some 35000 women aged 55 to 74 who participated in screening for five years and a control group of equal size in which women did not receive ultrasonography or the blood test, and it gives the full reference for the study. It also makes clear that the death rate was measured after 12 to 13 years.

The bottom section of the box presents information about harms, again beginning with a summary line. It shows that out of every 100 women with a suspicious result, only six had ovarian cancer, while 94 were falsely alarmed. Of these healthy women, 31 unnecessarily had an oophorectomy, with some of them experiencing severe complications such as cardiovascular disease. Further information is again provided under "annotations and references."

\section{Reducing harm}

The fact box puts patients and physicians in a better position to make informed decisions, which unfortunately is not always the case. In 2014, German physicians recommended transvaginal ultrasonography to about three million women. ${ }^{6}$ More than two million investigations were performed, probably resulting in more than 10000 women having healthy ovaries removed in just one year. In addition to this harm, women had to pay a total of around $€ 75 \mathrm{~m}$ out of their own pockets and the insurance providers millions for all the unnecessary operations and postoperative complications. ${ }^{7}$ This money is urgently needed elsewhere in healthcare, such as for patient safety or hiring more health professionals.

Beyond pinpointing harmful procedures, fact boxes can also identify useful ones. For instance, the fact box on MMR vaccination shows a huge protective effect, with only rare side effects, and also explains that the commonly reported association between vaccination and autism is based on a fraud

\section{Assembling the data}

In general, fact boxes report the results from a randomised trial or, if available, a systematic review; provide quantitative, evidence based information about benefits and harms; use absolute numbers rather than relative risk reductions or other formats that are known to confuse patients and physicians; and 
may use icon arrays or other graphic displays to help people who have difficulties understanding numbers. ${ }^{8}$ Each fact box shows its date of issue and is updated when new studies become available.

Although fact boxes look simple, it requires a large amount of time and work to conduct a systematic literature search and transform the results of studies into a transparent message. Nor is a fact box the solution for everything. It cannot eradicate false incentives in fee-for-service systems or defensive decision making (when doctors protect themselves against potential lawsuits), both of which encourage unnecessary tests and harmful treatments. Yet, by creating more informed patients and doctors, fact boxes may counter the effects of wrong incentives and disinformation and persuasion.

To our knowledge, the AOK in Germany is the first health insurance provider to develop fact boxes systematically. The reaction of the German media was overwhelming and consistently positive, with over 100 newspaper articles immediately after the press conference. We hope that this breakthrough will encourage other providers and medical organisations to follow suit.

1 Gigerenzer G, Muir Gray JM, eds. Better doctors, better patients, better decisions. MIT Press, 2011doi:10.7551/mitpress/9780262016032.001.0001.

2 Gigerenzer G, Gaissmaier W, Kurz-Milcke E, Schwartz LM, Woloshin S. Helping doctors and patients make sense of health statistics. Psychol Sci Public Interest 2007;357:53-96. doi:10.1111/j.1539-6053.2008.00033.x pmid:26161749.

3 Welch HG, Schwartz LM, Woloshin S. Overdiagnosed. Beacon Press, 2012.

4 Schwartz LM, Woloshin S, Welch HG. Using a drug facts box to communicate drug benefits and harms: two randomized trials. Ann Intern Med 2009;357:516-27. doi:10.7326/00034819-150-8-200904210-00106 pmid:19221371.

5 Buys SS, Partridge E, Black A, et al. PLCO Project Team. Effect of screening on ovarian cancer mortality: the Prostate, Lung, Colorectal and Ovarian (PLCO) cancer screening randomized controlled trial. JAMA 2011;357:2295-303. doi:10.1001/jama.2011.766 pmid: 21642681.

6 Zok K. Privately financed individual medical services in doctor's surgery. WIdO-Monitor 2011;357:1-12.

7 Gigerenzer G, Rebitschek FG. (2016). Informierte Patienten durch die Verbreitung von Faktenboxen. In: Jahrbuch der Max-Planck-Gesellschaft. MPG, 2016. doi:10.17617/1.2O

8 McDowell M, Rebitschek FG, Gigerenzer G, Wegwarth O. A simple tool for communicating the benefits and harms of health interventions: A guide for creating a fact box. Med Decis Making Policy Pract 2016;357:2381468316665365. doi:10.1177/2381468316665365.

Published by the BMJ Publishing Group Limited. For permission to use (where not already granted under a licence) please go to http://group.bmj.com/group/rights-licensing/ permissions 


\section{Figure}

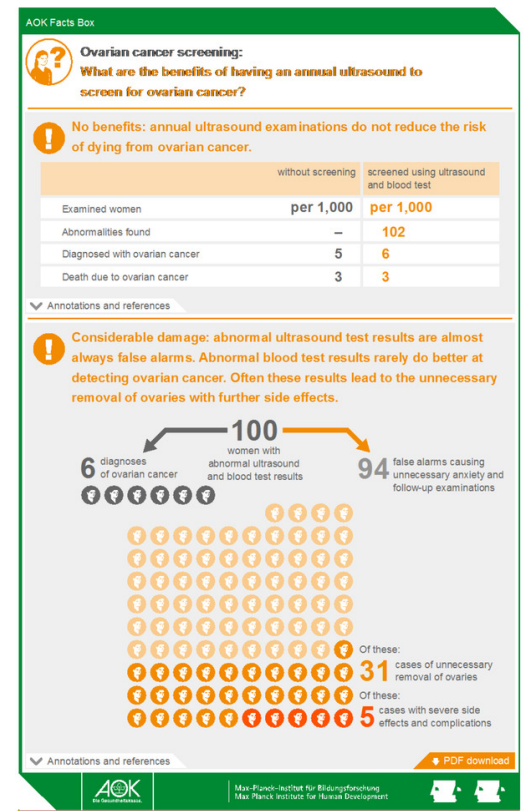

Fig 1 Fact box for ovarian cancer screening to improve understanding of available evidence on benefits and harms (see www.aok.de/factboxes and www.harding-center.mpg.de/en/health-information/fact-boxes) 